PROCEEDINGS OF THE

AMERICAN MATHEMATICAL SOCIETY

Volume 127, Number 10, Pages 2953-2960

S 0002-9939(99)04891-1

Article electronically published on April 23, 1999

\title{
ON THE LOCAL ASYMPTOTICS OF FABER POLYNOMIALS
}

\author{
IGOR E. PRITSKER
}

(Communicated by Albert Baernstein II)

Dedicated to Professor D. Gaier on the occasion of his seventieth birthday.

\begin{abstract}
We study a local asymptotics of the (generalized) Faber polynomials at the boundary of the associated domain, under certain mild smoothness conditions on the weight function and geometric conditions on the boundary. The main result exhibits how this asymptotics depends on the corners at the boundary. Its proof is based on the continuity properties of the VisserOstrowski quotient at the corners.
\end{abstract}

\section{INTRODUCTION AND MAIN RESULT}

Let $\Gamma$ be a simple bounded Jordan curve with the interior $G$ and the exterior $\Omega$. Clearly, $G$ and $\Omega$ are Jordan domains and $\infty \in \Omega$. By the Riemann mapping theorem, there exists a canonical conformal mapping $\Phi: \Omega \rightarrow D^{\prime}:=\{w:|w|>1\}$ normalized by the conditions $\Phi(\infty)=\infty$ and $\lim _{z \rightarrow \infty} \Phi(z) / z>0$. It is well known that $\Phi$ can be extended as a homeomorphism to the boundary $\Gamma$ (cf. [11, p. 24]), so that we have $\Phi: \bar{\Omega} \rightarrow \overline{D^{\prime}}$. Let $\Psi:=\Phi^{-1}$ be the inverse conformal mapping $\Psi: \overline{D^{\prime}} \rightarrow \bar{\Omega}$.

Faber polynomials were introduced by Faber [5] in connection with a generalization of the Taylor series for an analytic function in $G$. One of the equivalent definitions of the Faber polynomials $\left\{F_{n}(z)\right\}_{n=0}^{\infty}$, $\operatorname{deg} F_{n}=n$, is the following Laurent expansion (cf. [13] or [8])

$$
\frac{\Psi^{\prime}(w)}{\Psi(w)-z}=\sum_{n=0}^{\infty} \frac{F_{n}(z)}{w^{n+1}}, \quad z \in \bar{G}, \quad|w|>1,
$$

where the function on the left-hand side of (1.1) is called the generating function. Faber polynomials have found numerous applications in approximation theory (see, e.g., [8] and [13]) and in function theory (see references in [2]).

Let $g(z)$ be an analytic function in $\Omega$. Then, following [6], we define the generalized Faber polynomials $\left\{Q_{n}(z)\right\}_{n=0}^{\infty}, \operatorname{deg} Q_{n}=n$, as follows:

$$
\frac{g(\Psi(w)) \Psi^{\prime}(w)}{\Psi(w)-z}=\sum_{n=0}^{\infty} \frac{Q_{n}(z)}{w^{n+1}}, \quad z \in \bar{G}, \quad|w|>1 .
$$

Received by the editors November 4, 1997 and, in revised form, December 31, 1997.

1991 Mathematics Subject Classification. Primary 30C10, 30E15; Secondary 30E10, 30C35.

Key words and phrases. Faber polynomials, asymptotics, Visser-Ostrowski quotient.

Research supported in part by the National Science Foundation grant DMS-9707359.

(C)1999 American Mathematical Society 
Clearly, if $g(z) \equiv 1, z \in \Omega$, then $Q_{n}(z) \equiv F_{n}(z)$ for any $n=0,1,2, \ldots$ The generalized Faber polynomials are of great importance in the study of asymptotics of various extremal polynomials, such as orthogonal polynomials (cf. [17, 13, 14, 15] and [18]) and Chebyshev polynomials (cf. [7, 18]). The asymptotic properties of the generalized Faber polynomials themselves are crucial in the above studies. In addition to the already mentioned surveys on Faber polynomials, we refer to the monograph [16] exclusively devoted to this subject, which contains many other references.

An equivalent way to define Faber polynomials is to consider the polynomial part of the Laurent expansion of $\Phi^{n}(z)$ near $z=\infty$, i.e.,

$$
\Phi^{n}(z)=F_{n}(z)+E_{n}(z),
$$

where

$$
E_{n}(z)=O\left(\frac{1}{z}\right), \quad \text { as } z \rightarrow \infty .
$$

Correspondingly, we have for the generalized Faber polynomials that

$$
g(z) \Phi^{n}(z)=Q_{n}(z)+H_{n}(z)
$$

where

$$
H_{n}(z)=O\left(\frac{1}{z}\right), \quad \text { as } z \rightarrow \infty
$$

(see $[2,8]$ and [13], for example). A standard approach to the asymptotic representations of the (generalized) Faber polynomials is to show in (1.3) or (1.5) that

$$
E_{n}(z)=o(1) \text { or } H_{n}(z)=o(1), \quad \text { as } n \rightarrow \infty,
$$

for $z \in \Omega$. Furthermore, one can even strengthen this to include the boundary $\Gamma$, under certain smoothness assumptions on $\Gamma$ and the values of $g(z)$ on $\Gamma$, which is usually more difficult to accomplish. The asymptotic relation then takes the form

$$
Q_{n}(z)=g(z) \Phi^{n}(z)+o(1), \quad \text { as } n \rightarrow \infty,
$$

where $z \in \bar{\Omega}$ (cf. $[17,18]$ and [16]). We study a local version of the asymptotics for the generalized Faber polynomials on the boundary of $\Omega$, i.e., for $z \in \Gamma$. This allows us to considerably relax conditions imposed on $\Gamma$ and $g(z)$. In particular, we treat non-smooth cases of the function $g(z)$ and the boundary $\Gamma$, exhibiting the dependence of the asymptotics on the geometry of $\Gamma$, e.g., on the corners at the boundary.

For a continuous function $f$ on a compact set $K \subset \mathbb{C}$, we define its modulus of continuity $\omega(f, \delta)$ by (cf. $[10$, p. 43$]$ and $[11$, p. 46$])$ :

$$
\omega(f, \delta):=\sup \left\{\left|f\left(z_{1}\right)-f\left(z_{2}\right)\right|: \quad\left|z_{1}-z_{2}\right| \leq \delta \text { and } z_{1}, z_{2} \in K\right\} .
$$

It is not difficult to see that $\omega(f, \delta), \delta \in[0, \operatorname{diam}(K)]$, has the following properties (see $[10$, p. 43]):

(i) $\omega(f, \delta)$ is positive and increasing;

(ii) $\lim _{\delta \rightarrow 0} \omega(f, \delta)=0$;

(iii) if $K$ is convex, then $\omega(f, \delta)$ is subadditive, i.e.,

$$
\omega\left(f, \delta_{1}+\delta_{2}\right) \leq \omega\left(f, \delta_{1}\right)+\omega\left(f, \delta_{2}\right) .
$$


Any function $\omega(\delta), \delta \in[0, l]$, satisfying (i)-(iii), is called a modulus of continuity. Typical examples of moduli of continuity are $\omega(\delta)=\delta^{s}, 0<s \leq 1$, and $\omega(\delta)=$ $(-\log \delta)^{-t}, t>0$. A modulus of continuity is said to satisfy the Alper condition if

$$
\int_{0}^{\epsilon} \frac{\omega(x)}{x} \log \frac{1}{x} d x<\infty
$$

for some $\epsilon>0$.

A Jordan curve $\Gamma$ is said to be of class $C^{\omega}$ if it has a parametrization $\tau:[0,2 \pi) \rightarrow$ $\Gamma$ that is differentiable, with $\tau^{\prime}(x) \neq 0$ for any $x \in[0,2 \pi)$ and with $\tau^{\prime}(x)$ satisfying a continuity condition

$$
\left|\tau^{\prime}\left(x_{1}\right)-\tau^{\prime}\left(x_{2}\right)\right| \leq \omega\left(\left|x_{1}-x_{2}\right|\right), \quad x_{1}, x_{2} \in[0,2 \pi),
$$

where $\omega$ is a modulus of continuity. A Jordan arc is of class $C^{\omega}$ if it is a subarc of a Jordan curve of class $C^{\omega}$. $167])$.

Let $H^{\infty}(\Omega)$ be the Hardy space of bounded analytic functions in $\Omega$ (cf. [3, p.

Theorem 1.1. Let $\Gamma$ be a rectifiable Jordan curve and let $g(z) \in H^{\infty}(\Omega)$. For $z_{0} \in \Gamma$, assume that $\Gamma$ has an exterior angle of opening $\alpha \pi$ at $z_{0}, 0<\alpha \leq 2$, formed by $C^{\omega}$ arcs, where $\omega(x)$ satisfies the Alper condition (1.10). Suppose that the boundary values of $g(z)$ satisfy a Dini condition at $z_{0}$ :

$$
\left|g(z)-g\left(z_{0}\right)\right| \leq \lambda\left(\left|z-z_{0}\right|\right), \quad\left|z-z_{0}\right|<\delta, z \in \Gamma,
$$

with $\delta>0$ and a modulus of continuity $\lambda(x)$ such that

$$
\int_{0}^{\delta} \frac{\lambda(x)}{x} d x<\infty
$$

Then, the generalized Faber polynomials satisfy

$$
Q_{n}\left(z_{0}\right)=\alpha g\left(z_{0}\right) \Phi^{n}\left(z_{0}\right)+o(1), \quad \text { as } n \rightarrow \infty .
$$

It is often sufficient for applications to consider $\omega(x)=x^{\beta}$ and $\lambda(x)=x^{\gamma}$, with $0<\beta, \gamma \leq 1$.

Remark 1.2. The above theorem corrects an erroneous statement in [16, p. 102103] that the asymptotic relation (1.8) remains the same for the case of angles at the boundary. If $\alpha=1$, then we obtain the familiar asymptotics (1.8) from (1.14).

Remark 1.3. It is possible to estimate the rate at which $o(1)$ approaches zero, as $n \rightarrow \infty$, by imposing additional restrictions on $\Gamma$ and $g(z)$.

Remark 1.4. Assuming that $\bar{G}$ is convex and $\alpha \pi$ is the largest exterior angle at the boundary $\Gamma$, it was shown in [12] (see Satz 3 and its proof) that, for the case $g(z) \equiv 1$,

$$
\max _{z \in \bar{G}}\left|F_{n}(z)\right| \rightarrow \alpha, \quad \text { as } \quad n \rightarrow \infty .
$$

Moreover, the proof indicates that

$$
\left|F_{n}\left(z_{0}\right)\right| \rightarrow \alpha, \quad \text { as } \quad n \rightarrow \infty .
$$

However, Theorem 1.1 gives more precise information about boundary asymptotics of Faber polynomials under more general conditions. 


\section{VisSER-OSTROWSKI QUOTIENT IN CONFORMAL MAPPING}

Let $w_{0}:=\Phi\left(z_{0}\right)$, where $z_{0} \in \Gamma$. Define

$$
h(w):=\frac{w-w_{0}}{\Psi(w)-\Psi\left(w_{0}\right)} \Psi^{\prime}(w), \quad|w| \geq 1 .
$$

We remark that $h(w)$ is the well known Visser-Ostrowski quotient, which has been studied in many papers (see [11] and [9] for surveys). However, those researches were mainly devoted to the questions of the existence and the value of nontangential limit of $h(w)$, as $w \rightarrow w_{0}$. We are interested in the continuity properties of $h(w)$ near $w_{0}$.

Theorem 2.1. Let $\Gamma$ be a Jordan curve and let $z_{0} \in \Gamma$. Assume that $\Gamma$ has an exterior angle of opening $\alpha \pi$ at $z_{0}, 0<\alpha \leq 2$, formed by $C^{\omega}$ arcs, where $\omega(x)$ satisfies the Alper condition (1.10). Then $h(w)$ is continuous at $w_{0}=e^{i \theta_{0}}:=\Phi\left(z_{0}\right)$, with

$$
h\left(w_{0}\right):=\lim _{\theta \rightarrow \theta_{0}} h\left(e^{i \theta}\right)=\alpha .
$$

Furthermore, there exists $\epsilon>0$ such that

$$
\int_{-\epsilon}^{\epsilon} \frac{\left|h\left(e^{i\left(\theta_{0}+y\right)}\right)-\alpha\right|}{|y|} d y<\infty .
$$

Proof. Observe that $\omega(x)$ satisfies a Dini condition by (1.10):

$$
\int_{0}^{\delta} \frac{\omega(x)}{x} d x<\infty .
$$

Thus, the assumptions of Theorem 2.1 imply that we have a Dini-smooth corner at $z_{0}$, using the terminology of [11, p. 52]. An application of Theorem 3.9 of [11] immediately gives that the functions

$$
\frac{\Psi(w)-\Psi\left(w_{0}\right)}{\left(w-w_{0}\right)^{\alpha}} \text { and } \quad \frac{\Psi^{\prime}(w)}{\left(w-w_{0}\right)^{\alpha-1}}
$$

are continuous and different from zero in $\bar{U}$, where $U:=\left\{\left|w-w_{0}\right|<\delta\right\} \cap D^{\prime}$, for some $\delta>0$. Consequently, $h(w)$ is also continuous and different from zero in $\bar{U}$, but this is not sufficient for our purposes. We shall follow the proof of Theorem 3.9 of [11] to extract the order of continuity of $h(w)$ near $w_{0}$ from that argument.

First, we apply the transformation $z \rightarrow\left(z-z_{0}\right)^{1 / \alpha}$ to $\Psi(U)$ and straighten the angle at $z_{0}$. It is shown in the proof of Theorem 3.9 of [11] (see p. 53) that the arcs $C^{ \pm}$, forming the $\alpha \pi$ angle at $z_{0}$, are mapped onto one Dini-smooth arc containing point 0 , which is a part of the boundary of a Dini-smooth domain $H$ contained in the image of $\Psi(U)$ under the transformation $z \rightarrow\left(z-z_{0}\right)^{1 / \alpha}$. Furthermore, it is also shown that if $\tau_{ \pm}(x), 0 \leq x \leq b_{1}$, are the parametrizations of $C^{ \pm}$such that $\tau_{ \pm}(0)=z_{0}$ and $\tau_{ \pm}^{\prime}(x) \neq 0$, then the corresponding arc of $\partial H$ can be parametrized by

$$
v_{ \pm}(t)=\left(\tau\left(c_{ \pm} t^{\alpha}\right)-z_{0}\right)^{1 / \alpha}, \quad 0 \leq t \leq b_{2},
$$

where $c_{ \pm}=1 /\left|\tau_{ \pm}^{\prime}(0)\right|$, and

$$
\omega\left(v_{ \pm}^{\prime}, t\right) \leq M \omega\left(\tau_{ \pm}^{\prime}, c_{ \pm} t^{\alpha}\right),
$$


with $M>0$. Using the assumptions (1.11)-(1.10), we obtain from the above inequality that

$$
\omega\left(v_{ \pm}^{\prime}, t\right)=O\left(\omega\left(t^{\alpha}\right)\right), \quad \text { as } t \rightarrow 0 .
$$

Hence a parametrization of $\partial H$ in the whole, denoted by $v(t), t \in[0,2 \pi)$, can be chosen to satisfy

$$
\omega\left(v^{\prime}, t\right)=O\left(\omega\left(t^{\alpha}\right)\right), \quad \text { as } t \rightarrow 0 .
$$

Let $\psi$ be a conformal mapping of the unit disk $D:=\{w:|w|<1\}$ onto $H$, such that $\psi(1)=0$. Since $U \subset D^{\prime}$, there exists a conformal mapping $\phi$ of $D$ into $D^{\prime}$, with $\phi(1)=w_{0}$, which satisfies

$$
\psi(s)=\left(\Psi(\phi(s))-\Psi\left(w_{0}\right)\right)^{1 / \alpha}, \quad s \in D .
$$

Note that $\phi(s)$ is conformal in a neighborhood of $s=1$ by reflection principle. With the notation $w=\phi(s)$, we obtain

$$
\Psi(\phi(s))-\Psi\left(w_{0}\right)=\psi^{\alpha}(s)
$$

and

$$
\Psi^{\prime}(\phi(s))=\frac{\alpha \psi^{\alpha-1}(s) \psi^{\prime}(s)}{\phi^{\prime}(s)}
$$

It follows that

$$
h(\phi(s))=\alpha \frac{\phi(s)-\phi(1)}{(s-1) \phi^{\prime}(s)} \frac{s-1}{\psi(s)-\psi(1)} \psi^{\prime}(s), \quad s \in D .
$$

Clearly,

$$
h_{1}(s):=\frac{\phi(s)-\phi(1)}{(s-1) \phi^{\prime}(s)}
$$

is analytic in a neighborhood of $s=1$, so that $h_{1}(1)=1$. In view of this fact, our problem reduces to the study of

$$
h_{2}(s):=\frac{s-1}{\psi(s)-\psi(1)} \psi^{\prime}(s),
$$

which is the Visser-Ostrowski quotient for the conformal mapping $\psi(s)$ at $s=1$. Since $\psi$ maps $D$ onto the domain $H$ with Dini-smooth boundary, we obtain from Theorem 3.5 of [11] that $\psi^{\prime}(s)$ has a continuous extension to $\bar{D}$, such that

$$
\frac{\psi(s)-\psi(1)}{s-1} \rightarrow \psi^{\prime}(1) \neq 0, \quad \text { as } s \rightarrow 1, s \in \bar{D} .
$$

It follows immediately from the above and (2.6) that

$$
h_{2}(s) \rightarrow 1 \quad \text { and } \quad h(\phi(s)) \rightarrow \alpha, \quad \text { as } s \rightarrow 1, s \in \bar{D},
$$

proving (2.2). Moreover, since $\omega\left(v^{\prime}, t\right)$ satisfies the Alper condition by (1.10) and (2.5), we obtain from [1] that the modulus of continuity of $\psi^{\prime}(s)$ on $\bar{D}$ satisfies the Dini condition

$$
\int_{0}^{\epsilon} \frac{\omega\left(\psi^{\prime}, x\right)}{x} d x<\infty
$$

Let

$$
q(s):=\frac{\psi(s)-\psi(1)}{s-1}=\int_{0}^{1} \psi^{\prime}(1+t(s-1)) d t, \quad s \in \bar{D} .
$$


Then

$$
\begin{aligned}
\left|q\left(s_{1}\right)-q\left(s_{2}\right)\right| & \leq \int_{0}^{1}\left|\psi^{\prime}\left(1+t\left(s_{1}-1\right)\right)-\psi^{\prime}\left(1+t\left(s_{2}-1\right)\right)\right| d t \\
& \leq \omega\left(\psi^{\prime},\left|s_{1}-s_{2}\right|\right), \quad s_{1}, s_{2} \in \bar{D} .
\end{aligned}
$$

It is now easy to see from (2.7) and the above inequality that

$$
\left|h_{2}\left(s_{1}\right)-h_{2}\left(s_{2}\right)\right|=O\left(\omega\left(\psi^{\prime},\left|s_{1}-s_{2}\right|\right)\right), \quad s_{1}, s_{2} \in \bar{D} .
$$

Finally, we deduce from (2.6) that

$$
|h(\phi(s))-h(\phi(1))|=O\left(\omega\left(\psi^{\prime},|s-1|\right)\right), \quad \text { as } s \rightarrow 1, s \in \bar{D},
$$

so that

$$
\left|h(w)-h\left(w_{0}\right)\right|=O\left(\omega\left(\psi^{\prime},\left|w-w_{0}\right|\right)\right), \quad \text { as } w \rightarrow w_{0}, w \in \overline{D^{\prime}},
$$

because $\phi(s)$ is conformal in a neighborhood of $s=1$. Hence (2.3) follows at once from $(2.8)$.

\section{Proof of Theorem 1.1}

Let $w_{0}:=\Phi\left(z_{0}\right)$, where $z_{0} \in \Gamma$. Then one can write

$$
g(\Psi(w)) \frac{w-w_{0}}{\Psi(w)-\Psi\left(w_{0}\right)} \Psi^{\prime}(w)=\sum_{n=0}^{\infty} \frac{a_{n}\left(z_{0}\right)}{w^{n}}, \quad|w|>1,
$$

because the function on the left of (3.1) is analytic in $D^{\prime}$. Using this series expansion, we obtain the following representation for the generalized Faber polynomials (cf. [16], we give a simple proof below).

Lemma 3.1. For $z_{0} \in \Gamma$, we have

$$
Q_{n}\left(z_{0}\right)=\Phi^{n}\left(z_{0}\right) \sum_{k=0}^{n} \frac{a_{k}\left(z_{0}\right)}{\Phi^{k}\left(z_{0}\right)}, \quad n=0,1,2, \ldots
$$

Proof. From the definition of the generalized Faber polynomials (1.2), one can immediately see that

$$
\begin{aligned}
& g(\Psi(w)) \frac{w-w_{0}}{\Psi(w)-\Psi\left(w_{0}\right)} \Psi^{\prime}(w)=\left(w-w_{0}\right) \sum_{n=0}^{\infty} \frac{Q_{n}\left(z_{0}\right)}{w^{n+1}} \\
& =Q_{0}\left(z_{0}\right)+\sum_{n=1}^{\infty} \frac{Q_{n}\left(z_{0}\right)-w_{0} Q_{n-1}\left(z_{0}\right)}{w^{n}} .
\end{aligned}
$$

It follows by comparing the above series with (3.1) that

$$
a_{0}\left(z_{0}\right)=Q_{0}\left(z_{0}\right) \text { and } a_{n}\left(z_{0}\right)=Q_{n}\left(z_{0}\right)-w_{0} Q_{n-1}\left(z_{0}\right), \quad n \in \mathbb{N} .
$$

This gives a recurrence relation for the generalized Faber polynomials:

$$
Q_{0}\left(z_{0}\right)=a_{0}\left(z_{0}\right) \text { and } Q_{n}\left(z_{0}\right)=a_{n}\left(z_{0}\right)+\Phi\left(z_{0}\right) Q_{n-1}\left(z_{0}\right), \quad n \in \mathbb{N},
$$

which readily yields (3.2). 
Observe that the sum on the right of (3.2) is just a partial sum of the series (3.1) for $w=w_{0}=\Phi\left(z_{0}\right)$. Thus, if (3.1) converges at the boundary point $w_{0}=\Phi\left(z_{0}\right)$, then we obtain a certain asymptotics of the generalized Faber polynomials at $z=z_{0}$ from (3.2), because $\left|\Phi\left(z_{0}\right)\right|=1$. The questions to be answered here are about the conditions to insure convergence of the series in (3.1) and about the value of its sum at $w_{0}=\Phi\left(z_{0}\right)$, i.e., when this series converges to the boundary value of the function on the left of $(3.1)$ at $w_{0}=\Phi\left(z_{0}\right)$.

Proof of Theorem 1.1. Note that $\Psi^{\prime}(w) \in H^{1}\left(D^{\prime}\right)$ because $\Gamma$ is rectifiable (cf. [11, p. 134]). Since the function $h\left(e^{i \theta}\right)$ of $(2.1)$ is continuous at $\theta_{0}=\arg w_{0}$ by Theorem 2.1 , then $h\left(e^{i \theta}\right) \in L^{1}[0,2 \pi)$. Denoting

$$
f(w):=g(\Psi(w)) \frac{w-w_{0}}{\Psi(w)-\Psi\left(w_{0}\right)} \Psi^{\prime}(w)=g(\Psi(w)) h(w), \quad|w| \geq 1,
$$

we conclude that $f\left(e^{i \theta}\right) \in L^{1}[0,2 \pi)$, as $g\left(\Psi\left(e^{i \theta}\right)\right) \in L^{\infty}[0,2 \pi)$. It follows that $f(w) \in H^{1}\left(D^{\prime}\right)$ and that the series (3.1) is the Fourier series of the boundary values $f\left(e^{i \theta}\right)$ for $w=e^{i \theta}$ (cf. [3, p. 38]).

Observe that we have by (1.12) and (2.4):

$$
\begin{aligned}
\left|g\left(\Psi\left(e^{i \theta}\right)\right)-g\left(\Psi\left(e^{i \theta_{0}}\right)\right)\right| & \leq \lambda\left(\left|\Psi\left(e^{i \theta}\right)-\Psi\left(e^{i \theta_{0}}\right)\right|\right) \\
& \leq M_{1} \lambda\left(\left|e^{i \theta}-e^{i \theta_{0}}\right|^{\alpha}\right) \leq M_{2} \lambda\left(\left|\theta-\theta_{0}\right|^{\alpha}\right),
\end{aligned}
$$

where $\theta$ is close to $\theta_{0}$ and $M_{1}, M_{2}>0$. Furthermore, using (3.5), Theorem 2.1 and the last inequality, we conclude that $f\left(e^{i \theta}\right)$ is continuous at $\theta=\theta_{0}$, with

$$
f\left(e^{i \theta_{0}}\right)=\alpha g\left(\Psi\left(e^{i \theta_{0}}\right)\right)=\alpha g\left(z_{0}\right) .
$$

Applying Dini's test for $f\left(e^{i \theta}\right)$ at $\theta=\theta_{0}$, (see [4, p. 160]), we obtain by (1.13) and (2.3):

$$
\begin{aligned}
& \int_{0}^{\delta}\left|\frac{f\left(e^{i\left(\theta_{0}+y\right)}\right)+f\left(e^{i\left(\theta_{0}-y\right)}\right)}{2}-f\left(e^{i \theta_{0}}\right)\right| \frac{d y}{y} \\
\leq & \int_{-\delta}^{\delta} \frac{\left|f\left(e^{i\left(\theta_{0}+y\right)}\right)-f\left(e^{i \theta_{0}}\right)\right|}{2|y|} d y \\
\leq & M_{3} \int_{0}^{\delta} \frac{\lambda\left(y^{\alpha}\right)}{y} d y+M_{4} \int_{-\delta}^{\delta} \frac{\left|h\left(e^{i\left(\theta_{0}+y\right)}\right)-\alpha\right|}{|y|} d y<\infty,
\end{aligned}
$$

where $M_{3}, M_{4}>0$. Hence the Fourier series of $f\left(e^{i \theta}\right)$ in (3.1) converges to $f\left(e^{i \theta_{0}}\right)$ at $\theta=\theta_{0}$, i.e.,

$$
\lim _{n \rightarrow \infty} \sum_{k=0}^{n} \frac{a_{k}\left(z_{0}\right)}{w_{0}^{k}}=\lim _{n \rightarrow \infty} \sum_{k=0}^{n} \frac{a_{k}\left(z_{0}\right)}{\Phi^{k}\left(z_{0}\right)}=\alpha g\left(z_{0}\right) .
$$

Thus, (1.14) follows from (3.2) and (3.7) because $\left|\Phi\left(z_{0}\right)\right|=1$.

\section{ACKNowledgement}

The author would like to thank the referee for his valuable suggestions and, especially, for pointing out that the Alper condition (1.10) would be a natural smoothness condition on the arcs, for (2.3) to be valid. 


\section{REFERENCES}

[1] S. Ya. Alper, On the uniform approximation of functions of a complex variable in closed domains, Izv. Akad. Nauk SSSR, Ser. Mat. 19 (1955), 423-444. (in Russian) MR 17:729a

[2] J. H. Curtiss, Faber polynomials and the Faber series, Amer. Math. Monthly 78 (1971), 577-596. MR 45:2183

[3] P. L. Duren, Theory of $H^{p}$ Spaces, Academic Press, New York, 1970. MR 42:3552

[4] R. E. Edwards, Fourier Series, a Modern Introduction, vol. 1, Springer-Verlag, New York, 1979. MR 80j: 42001

[5] G. Faber, Über polynomische Entwicklungen, Math. Ann. 57 (1903), 389-408.

[6] G. Faber, Über polynomische Entwicklungen II, Math. Ann. 64 (1907), 116-135.

[7] G. Faber, Über Tschebyscheffsche Polynome, J. Reine Angew. Math. 150 (1920), 79-106.

[8] D. Gaier, Lectures on Complex Approximation, Birkhäuser, Boston, 1987. MR 88i:30059b

[9] C. Gattegno and A. Ostrowski, Représentation Conforme à la Frontière: Domaines Particuliers, Mém. Sci. Math., vol. 110, Paris, 1949. MR 11:426a

[10] G. G. Lorentz, Approximation of Functions, Chelsea Publ. Co., New York, 1986. MR 88j: 41001

[11] Ch. Pommerenke, Boundary Behaviour of Conformal Maps, Springer-Verlag, Berlin, 1992. MR 95b:30008

[12] Ch. Pommerenke, Über die Faberschen Polynome schlichter Funktionen, Math. Z. 85 (1964), 197-208. MR 29:6028

[13] V. I. Smirnov and N. A. Lebedev, Functions of a Complex Variable: Constructive Theory, MIT Press, Cambridge, 1968. MR 37:5369

[14] P. K. Suetin, V. A. Steklov's problem in the theory of orthogonal polynomials, J. Soviet Math. 12 (1979), 631-682.

[15] P. K. Suetin, Polynomials Orthogonal over a Region and Bierberbach Polynomials, Amer. Math. Soc., Providence, 1974. MR 57:3732b

[16] P. K. Suetin, Series in Faber Polynomials, Nauka, Moscow, 1984. (in Russian)

[17] G. Szegö, Orthogonal Polynomials, Amer. Math. Soc., Providence, 1975. MR 51:8724

[18] H. Widom, Extremal polynomials associated with a system of curves in the complex plane, Adv. Math. 3 (1969), 127-232.

Department of Mathematics, Case Western Reserve University, 10900 Euclid Avenue, Cleveland, Ohio 44106-7058

E-mail address: iep@po.cwru.edu 